\title{
Resection of Bilateral C1 Neurofibromas Using a Unilateral Modified Far-Lateral Approach
}

\author{
Jonathan Liu $^{1}$, Indro Chakrabarti ${ }^{2}$, Michael W. McDermott ${ }^{3}$ \\ 1. Neurological Surgery, University of California, Davis 2. Neurological Surgery, Kaiser Foundation \\ Medical Center 3. Department of Neurological Surgery, University of California, San Francisco
}

$\square$ Corresponding author: Jonathan Liu, jjliu3@gmail.com

Disclosures can be found in Additional Information at the end of the article

\section{Abstract}

Resection of foramen magnum lesions through a lateral approach can be technically challenging. Surgical avenues to foramen magnum lesions include a suboccipital craniectomy with C1 laminectomy, a modified far lateral approach with little condylar drilling, or via an anterior approach. The authors report their surgical experience in the treatment of a 36-yearold male with bilateral C1 dumbbell neurofibromas using a unilateral modified far-lateral approach (no condylar drilling performed). The unilateral modified far-lateral approach allowed for an efficient and safe corridor to this patient's bilateral foramen magnum tumors.

Categories: Neurosurgery

Keywords: neurofibroma, foramen magnum tumors, meningioma, far-lateral approach

\section{Introduction}

Most intradural extramedullary tumors occupying the ventral or ventrolateral cervical spinal cord and foramen magnum are meningiomas and neurofibromas [1-2]. Other pathology in this area includes schwannomas of the lower cranial nerves, chordomas, neurenteric cysts, and hemangioblastomas [3]. Large dumbbell-shaped C1 schwannomas have also been described by Helms, et al. as a rare pathology originating from the now elucidated sensory contribution to the $\mathrm{C} 1$ nerve root [4].

The foramen magnum is anatomically defined as the region extending from the junction of the middle third of the clivus to the anterior arch of $\mathrm{C} 1$ and the odontoid. Lateral to the foramen magnum are the occipital condyles, atlas lateral mass, and the jugular tubercle [5]. Surgical avenues to foramen magnum lesions include a suboccipital craniectomy and C1 laminectomy, a modified far lateral approach with little condylar drilling [2], or an anterior approach using a

Received 09/24/2014 Review began 09/29/2014 Review ended 11/14/2014 Published 11/16/2014

C) Copyright 2014

Liu et al. This is an open access article distributed under the terms of the Creative Commons Attribution License CC-BY 3.0., which permits unrestricted use, distribution, and reproduction in any medium, provided the original author and source are credited. transoral or transcervical route [6]. The anterior approaches have its disadvantages, including destabilization of the cervical spine, poor visibility lateral to the vertebral arteries, infection, and potential cerebrospinal fluid leakage [6].

Removal of such lesions has largely been supplanted by lateral craniocervical approaches. The extreme lateral approach provides a shallow and direct visualization of the anterior craniocervical junction without significant brain retraction [3]. The craniotomy involves posterolateral bone resection that includes the lateral rim of the foramen magnum and posterior occipital condyle [3]. Antero-lateral and ventral foramen magnum meningiomas of the foramen magnum, as well as schwannomas/neurofibromas of lower cranial or upper cervical 
nerves, can be accessed with far-lateral and modified approaches. While the literature has described removal of neurofibromas from the ventral craniocervical junction, this is the first description of resecting bilateral anterior craniocervical dumbbell neurofibromas through a unilateral modified far lateral approach.

\section{Case Presentation}

A 36-year-old male with a history of Neurofibromatosis Type 1 has been closely monitored for growth of multiple peripheral nerve neurofibromas. Prior to surgery for the craniocervical neurofibromas, he underwent C3-C5 laminectomies for removal of very large ventral kissing neurofibromas at the $\mathrm{C} 3-\mathrm{C} 4$ levels. He was symptomatic from these tumors with progressive dysequilibrium, hand numbness, and weakness. He underwent a complete resection of the intradural components at this level. The extradural components at C3-4 were initially followed conservatively. However, when serial imaging of the extradural components at C3-4 demonstrated enlarging tumors, they were treated with radiosurgery.

Serial imaging of his spinal axis revealed three additional areas of concern. These included a left T4-T5 foraminal lesion with dorsal extradural extension to the T5 level, L4 bilateral kissing neurofibromas, and bilateral neurofibromas of the $\mathrm{C} 1$ nerve root. Both the thoracic and lumbar lesions were conservatively managed and remained asymptomatic.

MRI with gadolinium of the cervical spine demonstrated bilateral C1 neurofibromas, larger on the left (Figure 1A-1B). 


\section{Cureus}
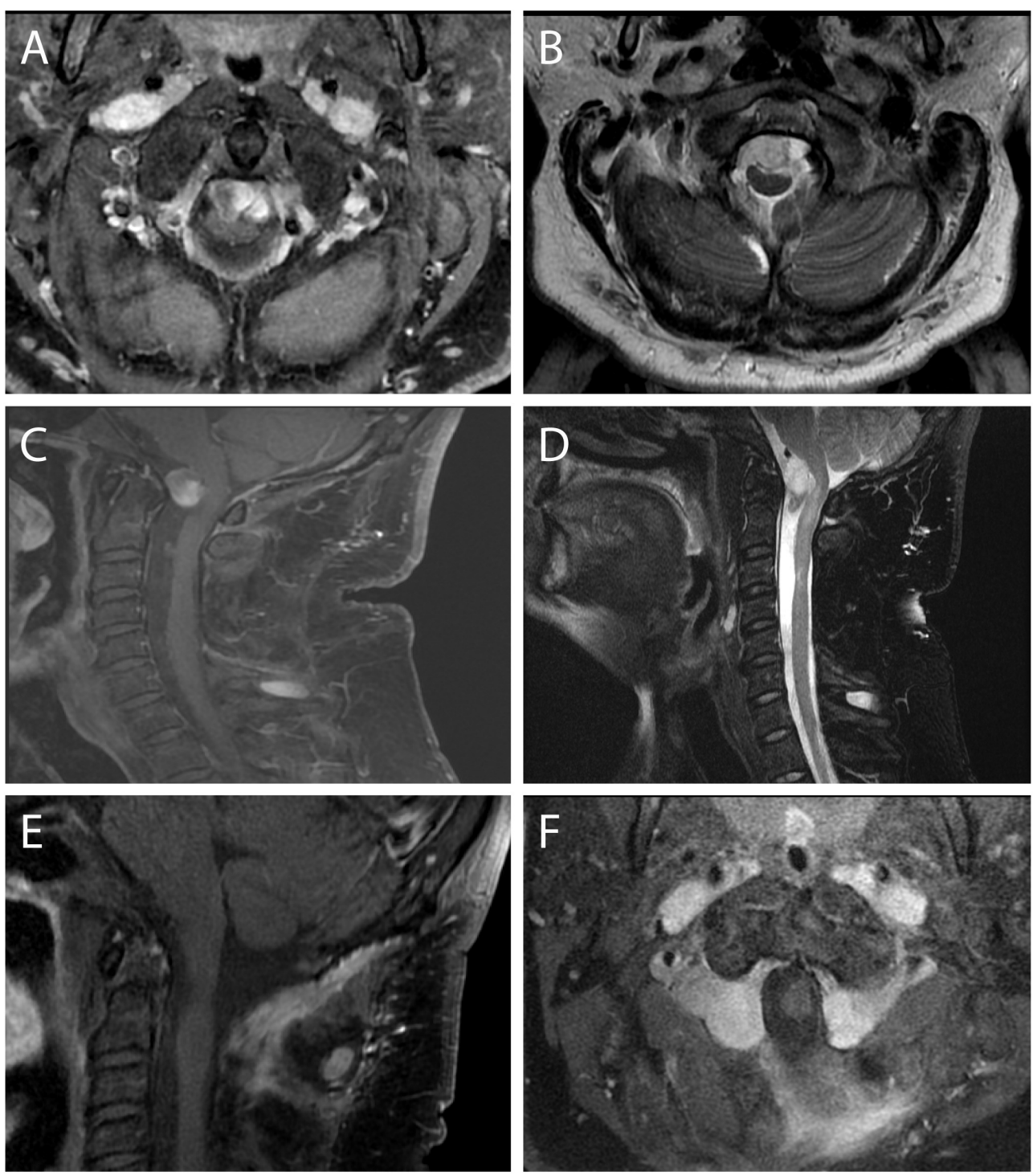

\section{FIGURE 1: Preoperative and Postoperative MRI's}

Preoperative axial MRI (A) T1 + gadolinium and T2 (B) showing bilateral cervicomedullary neurofibromas. Preoperative sagittal MRI (C) T1 + Gadolinium showing dorsal displacement of the cervicomedullary junction and T2 (D) showing spinal cord signal change. Postoperative sagittal MRI (E) T1 + gadolinium showing decompression of the spinal cord after resection of the intradural neurofibromas. Postoperative axial MRI (F) T1 + gadolinium.

The large left dumbbell shaped heterogeneously enhancing mass had both intradural and extradural components at the $\mathrm{C} 1$ nerve root. Serial imaging demonstrated continued enlargement of the intradural component with increasing mass effect on the dorsal medullary cord. The patient also became symptomatic from these lesions, with progressive symptoms of disequilibrium. The intradural component measured approximately $2 \mathrm{~cm}$ in the craniocaudal dimension and $1.2 \mathrm{~cm}$ in the anterior/posterior dimension. The intradural component caused significant canal stenosis with compression of the craniocervical junction and dorsal displacement of the cervical cord.

In addition, a smaller right $\mathrm{C} 1$ dumbbell lesion was also seen with a smaller intradural compressive component. Over a three-year period, the lesions at the foramen magnum showed 
a slight interval increase in size with worsening dorsal displacement of the spinal cord (Figure $1 C-1 D)$.

The combination of young age and progressive tumor growth prompted a debulking procedure followed by adjuvant radiosurgery. Early surgery would also circumvent neurologic compromise from progressive spinal cord and brainstem compression. Written informed consent was obtained to perform the surgical procedure on this patient but waived for the writing of this case report.

Postoperatively the patient did not suffer any new neurological deficits. MRI of the cervical spine after resection demonstrated decompression of the cervicomedullary junction with gross total resection of the intradural tumor (Figure $1 E-1 F$ ).

\section{Surgical procedure}

In the operating room, the patient was first placed in lateral position for placement of a lumbar subarachnoid drain. The patient was then placed in three-quarters prone position with the left side up on the operating room table with an underlying axillary roll. In the modified park-bench position, the right arm was padded and supported in a sling, hanging freely off the operating table.

The patient's head was then secured in a Mayfield headholder, followed by registration for neuronavigation. After the patient was adequately padded and secured, the ipsilateral shoulder was taped and pulled towards the feet to exaggerate the corridor between the patient's head and shoulder. A hockey stick shaped incision was then fashioned, beginning at the ipsilateral mastoid tip, continuing above the superior nuchal line, and curving to the midline, connecting to the previous midline cervical scar. After incision, a myocutaneous flap was reflected laterally, leaving a muscle cuff at the nuchal line. Subperiosteal dissection was carried down to the C2 level along the midline raphe and laterally to the mastoid tip.

A suboccipital craniectomy was then performed using a high-speed drill. The transverse and sigmoid sinuses were identified and skeletonized down to the jugular bulb. The craniectomy was then carried to the midline and down through the lip of the foramen magnum. Attention was then turned to completion of the C1 laminectomy and C2 hemilaminectomy. The C1 laminectomy was performed using a high-speed drill and up-biting Kerrison rongeur. The sulcus arteriosus was identified to ensure preservation of the vertebral artery. After the posterior arch of $\mathrm{C} 1$ was removed, the left hemilamina of $\mathrm{C} 2$ was removed out to the level of the pedicle. The occipital condyle was left intact, hence a "modified" far lateral.

The dura was subsequently opened in a curvilinear fashion starting laterally at the sigmoid sinus and curving towards midline down to the cervicomedullary junction. The dural incision was then carried out medially at the inferior pole to help expose the contralateral lesion. The dural edges were then retracted, followed by CSF drainage from the cervical subarachnoid space.

The remainder of the surgery was done under high magnification. Immediately upon opening the dura, tumor was clearly seen deviating the spinal cord dorsally.

The compressive nature of the left-sided neurofibroma was further appreciated after extensive arachnoid dissection (Figure $2 A$ ). 


\section{Cureus}
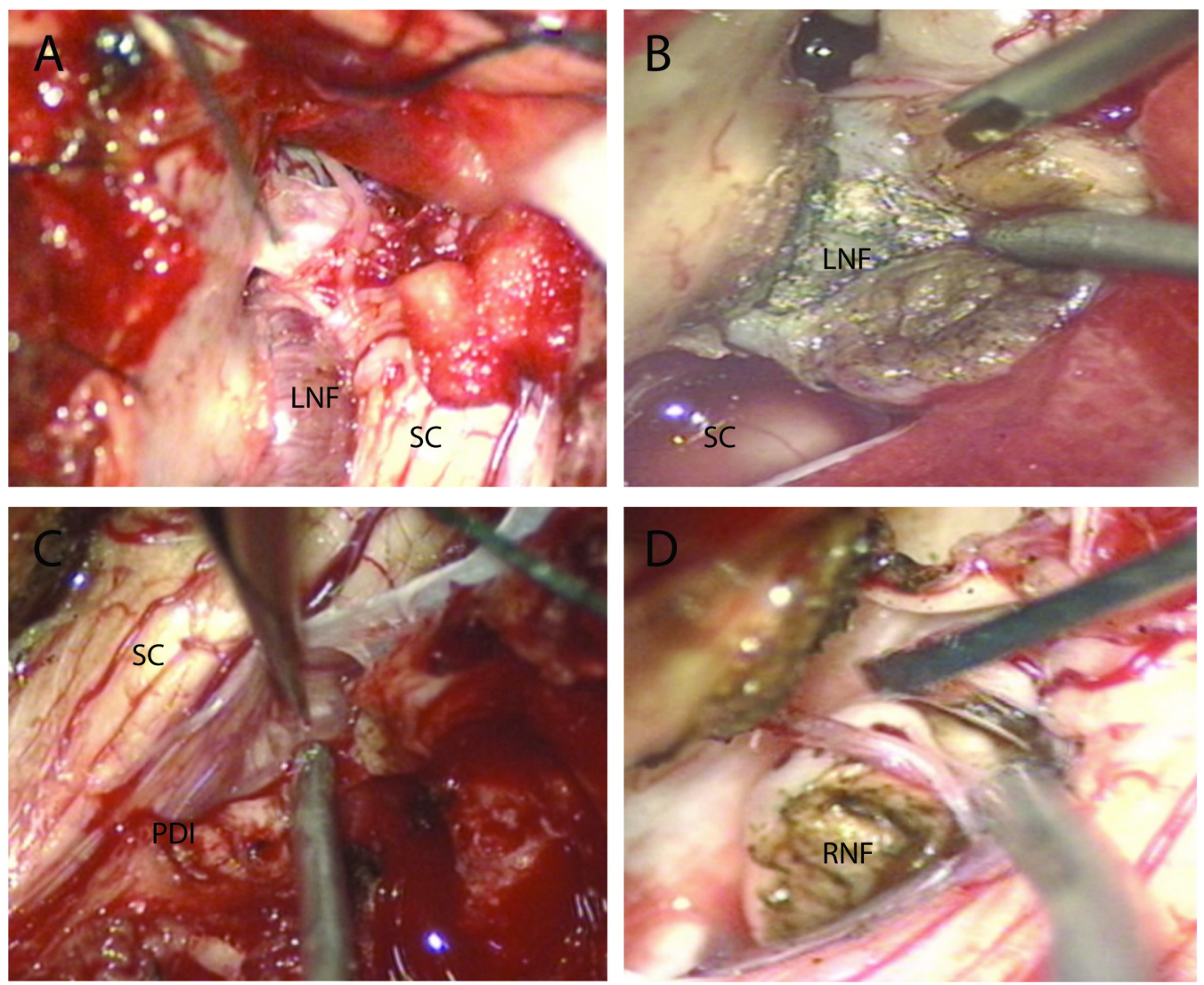

\section{FIGURE 2: Intraoperative Photographs}

(A) Intraoperative photograph showing dorsal displacement of the spinal cord by the left-sided neurofibroma. (B) Debulking of left-sided tumor using carbon dioxide laser. (C) Approaching right-tumor with a perpendicular dural incision towards the right $\mathrm{C} 1$ nerve root. (D) Removal of right-sided neurofibroma from the ventral spinal cord. LNF=left-sided neurofibroma; $\mathrm{PDI}=$ perpendicular dural incision; RNF=right-sided neurofibroma; $\mathrm{SC}=$ spinal cord

At this point, the tumor was seen deviating the cord to the right and posteriorly. The dentate ligaments were cut to improve maneuverability. The left $\mathrm{C} 1$ rootlet was draped over the tumor, which was bipolared and sacrificed. A cottonoid was then placed between the tumor and the rostrally situated vertebral artery.

Once hemostasis was achieved, the tumor resection started. The left-sided intradural tumor was debulked and removed piecemeal using a combination of bipolar, suction, and carbon dioxide laser (Figure 2B).

Once the left-sided tumor was adequately debulked, the tumor capsule could be lifted off the spinal cord, allowing for visualization of the right-sided intradural tumor. To enhance the exposure of the contralateral side, the dura was further opened perpendicular to the initial dural incision towards the contralateral $\mathrm{C} 1$ nerve root to better expose the right-sided tumor (Figure 2C).

The dissection proceeded intradurally to include further arachnoid dissection and cutting the contralateral dentate ligament. A corridor was then established ventral to the spinal cord allowing for visualization of the right-sided tumor as it exited the neural foramina. The tumor pedicle was amputated at the foramina dorsally, and again debulked using the carbon dioxide laser. Once debulked, the remaining tumor could be pulled out from the ventral side of the 
brainstem (Figure 2D).

Once bilateral intradural tumors were removed, the spinal cord and brainstem were well decompressed. The dura was then closed using cellulose dural matrix.

Once the dura was closed, the left-sided extradural component was debulked using the carbon dioxide laser and scissors. The wound was then closed in multiple layers. All motor evoked potentials and somatosensory evoked potentials were unchanged throughout the operation. There were also no abnormal electromyographic changes from the lower cranial nerves after tumor debulking.

\section{Discussion}

The lateral suboccipital approach for resection of foramen magnum tumors has been modified by many surgeons to help facilitate improved visualization of the ventral cervicomedullary junction with limited retraction. The first variation was the far lateral approach that included inferolateral bone removal of the foramen magnum towards the condylar fossa and partial removal of the $\mathrm{C} 1 \mathrm{arch}$ [7]. This approach was then further modified to include condylar resection for tumors when more ventral exposure was needed. This technique has been described and popularized by Sen and Sekhar in 1990 as a means to facilitate the removal of intradural extramedullary tumors of the foramen magnum and cervical spine when the posterior approach had failed [6].

Although many surgeons have adopted the above techniques, not all tumors of the foramen magnum require the same amount of bone work. According to Nanda, et al., there is no data in the literature that supports the superiority of condylar resection during a far lateral approach [7]. Rather the ability to achieve a complete tumor resection is more influenced by its location, extension, and intrinsic characteristics. Sanai and McDermott have described a modified far lateral approach where the occipital condyle is not drilled for resection of large posterior fossa meningiomas. This approach allowed for safe and often complete tumor resection while avoiding the risks and morbidity associated with condylar resection [2].

With the above considerations preoperatively, we describe the safe removal of bilateral C1 dumbbell neurofibromas using a modified far lateral approach without condylar resection. While this method may carry increased risk of operative or neurologic injury, we were able to first safely expose and debulk the left-sided tumor, followed by extension of our dural opening perpendicular to the initial cut in the direction of the contralateral nerve root. Although we describe the feasibility of a modified far-lateral technique for this particular case, this may not be the best approach for all bilateral C1 lesions. Dorsal displacement of the spinal cord created a safe ventral corridor that facilitated the safe removal of the contralateral tumor with minimal surgical retraction of the cervicomedullary junction. We were able to decompress the cervicomedullary junction through a unilateral intradural approach. Postoperatively, this patient did not suffer lower cranial nerve deficits or new focal neurological deficits.

\section{Conclusions}

We have presented the first case of safe removal of bilateral C1 neurofibromas with laser assistance using a unilateral modified far lateral approach. This is an efficient technique to maximize resection of bilateral foramen magnum tumors when clear planes can be distinguished between the spinal cord and tumor. The ability to achieve resection of bilateral lesions minimizes patient morbidity by possibly eliminating the need for bilateral surgical approaches to these formidable lesions. 


\section{Additional Information \\ Disclosures}

Human subjects: Consent was obtained by all participants in this study. Conflicts of interest: In compliance with the ICMJE uniform disclosure form, all authors declare the following:

Payment/services info: All authors have declared that no financial support was received from any organization for the submitted work. Financial relationships: All authors have declared that they have no financial relationships at present or within the previous three years with any organizations that might have an interest in the submitted work. Other relationships: All authors have declared that there are no other relationships or activities that could appear to have influenced the submitted work.

\section{References}

1. Dodge HW Jr, Gottlieb CM, Love JG: Benign tumors at the foramen magnum; surgical considerations. J Neurosurg. 1956, 13:603-17.

2. Sanai N, McDermott MW: A modified far-lateral approach for large or giant meningiomas of the posterior fossa. J Neurosurg. 2010, 112:907-12. 10.3171/2009.6.JNS09120

3. Menezes AH: Surgical approaches: Postoperative care and complications "posterolateral-far lateral transcondylar approach to the ventral foramen magnum and upper cervical spinal canal". Childs Nerv Syst. 2008, 24:1203-7. 10.1007/s00381-008-0597-5

4. Helms J, Michael LM 2nd: Large dumbbell-shaped c1 schwannoma presenting as a foramen magnum mass. J Neurol Surg Rep. 2012, 73:32-6. 10.1055/s-0032-1311757

5. Gupta SK, Sharma BS, Khosla VK, Mathuria SN, Pathak A, Tewari MK: Far lateral approach for foramen magnum lesions. Neurol Med Chir (Tokyo). 2000, 40:48-52.

6. Sen CN, Sekhar LN: An extreme lateral approach to intradural lesions of the cervical spine and foramen magnum. Neurosurg. 1990, 27:197-204.

7. Nanda A, Vincent DA, Vannemreddy PS, Baskaya MK, Chanda A: Far-lateral approach to intradural lesions of the foramen magnum without resection of the occipital condyle. J Neurosurg. 2002, 96:302-9. 